\title{
Brief Announcement: Variants of Approximate Agreement on Graphs and Simplicial Complexes
}

\author{
Jérémy Ledent \\ jeremy.ledent@strath.ac.uk \\ MSP Group, University of Strathclyde \\ Glasgow, United Kingdom
}

\begin{abstract}
Approximate agreement is a weaker version of consensus where two or more processes must agree on a real number within a distance $\varepsilon$ of each other. Many variants of this task have been considered in the literature: continuous or discrete ones; multi-dimensional ones; as well as agreement on graphs and other spaces. We focus on two variants of approximate agreement on graphs, edge agreement and clique agreement. We show that both tasks arise as special cases of a more general, higher-dimensional, approximate agreement task, where the processes must agree on the vertices of a simplex in a given simplicial complex. This new point of view gives rise to a novel topological perspective on the solvability of clique agreement.
\end{abstract}

\section{CCS CONCEPTS}

- Theory of computation $\rightarrow$ Distributed algorithms.

\section{KEYWORDS}

distributed computing; approximate agreement; simplicial complex

\section{ACM Reference Format:}

Jérémy Ledent. 2021. Brief Announcement: Variants of Approximate Agreement on Graphs and Simplicial Complexes. In Proceedings of the 2021 ACM Symposium on Principles of Distributed Computing (PODC '21), July 2630, 2021, Virtual Event, Italy. ACM, New York, NY, USA, 4 pages. https: //doi.org/10.1145/3465084.3467946

\section{INTRODUCTION}

Approximate agreement [6] is a weaker variant of the consensus task, which is solvable in the wait-free read/write registers model Many variants of approximate agreement have been considered, with a similar structure. Fix a finite number $n$ of processes $\left(P_{i}\right)_{i \in[n]}$. Each process $P_{i}$ starts the computation with an input value $x_{i} \in \mathcal{V}$, where $\mathcal{V}$ is some set of values. At the end of the computation, each process decides on an output value $y_{i} \in \mathcal{V}$, subject to some taskspecific constraints, known as validity and agreement. Validity restricts the set of possible outputs depending on the set $\left\{x_{i} \mid i \in[n]\right\}$ of inputs values; and agreement says that the output values chosen by the processes should be "close from each other". Thus, the set $\mathcal{V}$ usually carries some additional structure (e.g. a metric space) in order to have a notion of "closeness" of the values $y_{i}$.

Permission to make digital or hard copies of part or all of this work for personal or classroom use is granted without fee provided that copies are not made or distributed for profit or commercial advantage and that copies bear this notice and the full citation on the first page. Copyrights for third-party components of this work must be honored

For all other uses, contact the owner/author(s).

PODC '21, fuly 26-30, 2021, Virtual Event, Italy

(c) 2021 Copyright held by the owner/author(s)

ACM ISBN 978-1-4503-8548-0/21/07.

https://doi.org/10.1145/3465084.3467946
$\varepsilon$-Approximate agreement. Fix a real number $\varepsilon>0$. The set of values is $\mathcal{V}=[0,1]$, the set of real numbers between 0 and 1 . The goal is to decide outputs $y_{i} \in[0,1]$ that are within distance $\varepsilon$. This is formalized by two conditions, validity and agreement:

- Validity: for all $i \in[n], \min _{j \in[n]} x_{j} \leq y_{i} \leq \max _{j \in[n]} x_{j}$.

- Agreement: for all $i, j \in[n],\left|y_{i}-y_{j}\right| \leq \varepsilon$.

For the analysis of distributed algorithms, it is often easier to manipulate discrete data; so rather than working with the interval $[0,1]$, it is handy to use the following discrete reformulation.

$N$-Approximate agreement. Fix some natural number $N \in \mathbb{N}_{>0}$, and take the set of values $\mathcal{V}=\{0,1,2, \ldots, N\}$. Processes start with input values $x_{i} \in \mathcal{V}$ and must decide outputs $y_{i} \in \mathcal{V}$ such that:

- Validity: for all $i \in[n], \min _{j \in[n]} x_{j} \leq y_{i} \leq \max _{j \in[n]} x_{j}$.

- Agreement: for all $i, j \in[n],\left|y_{i}-y_{j}\right| \leq 1$.

Thus, this is the same as the previous task for $\varepsilon=\frac{1}{N}$, where the processes are restricted to choose values of the form $\frac{k}{N}, 0 \leq k \leq N$.

The $N$-approximate agreement task can also be viewed as a special case of a graph agreement task. Let $\mathcal{G}$ be the graph with set of vertices $\mathcal{V}=\{0,1,2, \ldots, N\}$, and edges of the form $\{k, k+1\}$ for each $0 \leq k<N$. The processes are given vertices of $\mathcal{G}$ as inputs, and must decide on a vertex of $\mathcal{G}$ so that the set of all outputs is an edge of $\mathcal{G}$. There are actually several ways to generalize this task to a general graph $\mathcal{G}$. In the two tasks below, we fix a finite graph $\mathcal{G}$, and let the set of values $\mathcal{V}$ be the set of vertices of $\mathcal{G}$.

Edge agreement. See e.g. [5] and [2].

- Validity: if all input vertices are equal, then every process must output this vertex; if the input vertices span an edge, then every process must output a vertex of that edge.

- Agreement: the set of output values $\left\{y_{i} \mid i \in[n]\right\}$ is either a vertex or an edge of $\mathcal{G}$.

Clique agreement. See e.g. [2], [12] and [3].

- Validity: if the set of inputs $X=\left\{x_{i} \mid i \in[n]\right\}$ is a clique of $\mathcal{G}$, then every output $y_{i}$ must be in $X$.

- Agreement: the set of outputs $\left\{y_{i} \mid i \in[n]\right\}$ is a clique of $\mathcal{G}$.

In the literature, edge agreement is known as graph convergence in [5] and edge gathering in [2]. Clique agreement is called 1 -gathering in [2], and variants of it (with a stronger validity condition) are monophonic approximate agreement in [12], and graphical approximate agreement in [3].

In the wait-free shared memory model, edge agreement is solvable for $n \geq 3$ processes if and only if the graph $\mathcal{G}$ is a tree [5]. For clique agreement, a precise characterization of solvability is not known yet. In [12], an algorithm is given to solve clique agreement on chordal graphs for $n \geq 3$ processes; and in [3] on nicely bridged 
graphs. Clique agreement is unsolvable on large cycles [5], and on graphs satisfying a so-called lower bound labelling condition [3].

Many other variants of approximate agreement have been studied. One of them is multi-dimensional approximate agreement [11], where processes takes values in the $m$-dimensional Euclidean space $\mathcal{V}=\mathbb{R}^{m}$. As in the 1-dimensional case, processes must decide output values within a distance $\varepsilon$ of each other. One could imagine similar tasks on various topological spaces (e.g. on a sphere or a torus), but most of them would be unsolvable. Indeed, Herlihy and Shavit [9] have shown that in the case where the space $\mathcal{V} \subseteq \mathbb{R}^{m}$ may contain holes, the corresponding approximate agreement task is not wait-free solvable.

Other notable examples of approximate agreement tasks include barycentric agreement [7], which is a discrete counterpart of multidimensional agreement; rendezvous tasks [10], loop agreement [8]; and more generally abstract approximate agreement [12] where $\mathcal{V}$ is an abstract convexity space.

Distributed computing model. Most of this paper is concerned with comparing task specifications, which is independent from the model used to solve the task. When we mention solvability, it will usually be in the wait-free shared memory model.

Contributions. Consider the simplex agreement task, which is a mild generalization of barycentric agreement ${ }^{1}$. Fix a simplicial complex $\mathcal{K}$, and let $\mathcal{V}$ be the vertices of $\mathcal{K}$. Processes are given input values $x_{i} \in \mathcal{V}$ and must decide outputs $y_{i} \in \mathcal{V}$ such that:

- Validity: if the set of input values $X=\left\{x_{i} \mid i \in[n]\right\}$ is a simplex of $\mathcal{K}$ then every output $y_{i}$ must be in $X$.

- Agreement: the set of outputs values $\left\{y_{i} \mid i \in[n]\right\}$ must be a simplex of $\mathcal{K}$.

We show that both edge agreement and clique agreement arise as special cases of this simplex agreement task. In particular, clique agreement on a graph $\mathcal{G}$ is equivalent to simplex agreement on the complex of cliques of $\mathcal{G}$, that we denote by $\kappa(\mathcal{G})$. Thus, we argue that, despite being formulated on a graph, clique agreement is in fact of a multi-dimensional nature. More precisely, the dimension of the associated simplicial complex $\kappa(\mathcal{G})$ is related to the clique number $\omega(\mathcal{G})$ of the graph by the formula: $\operatorname{dim}(\kappa(\mathcal{G}))+1=\omega(\mathcal{G})$.

This topological point of view sheds a new light on the solvability of clique agreement. Indeed, the chordality of a graph is known to be related to the contractibility of its complex of cliques [1] Thus, we conjecture that clique agreement is solvable on a graph $\mathcal{G}$ precisely when $\kappa(\mathcal{G})$ is contractible. This would connect nicely with the continuous case, where multi-dimensional $\varepsilon$-agreement in a subset $\mathcal{V} \subseteq \mathbb{R}^{m}$ is known to be unsolvable if $\mathcal{V}$ contains a hole of radius larger than $\varepsilon$ [9].

Plan. In Section 2, we recall some basic definitions on graphs and simplicial complexes, and state the relationship between a graph and its complex of cliques as an adjunction between categories. Then in Section 3, we show that both edge and clique agreement are particular instances of the simplex agreement task. We highlight the relevance of this fact by looking at known results on graph agreement tasks through the lens of topology.

\footnotetext{
${ }^{1}$ Barycentric agreement is recovered when the simplicial complex $\mathcal{K}$ is chosen to be the iterated barycentric subdivision of a standard $n$-simplex.
}

\section{ADJUNCTIONS BETWEEN GRAPHS AND SIMPLICIAL COMPLEXES}

We quickly recall the standard definitions of graphs and simplicial complexes. Note that our graphs are non-directed and simple, i.e., without self-loops or parallel edges. The main purpose of this section is to explain how every graph $\mathcal{G}$ can be turned into a simplicial complex $\kappa(\mathcal{G})$, called the complex of cliques. This complex $\kappa(\mathcal{G})$ is canonical, in the sense of Proposition 1. We use the language of category theory to formally study $\kappa$, but a reader unfamiliar with those notions should not worry: understanding the definition of $\kappa(\mathcal{G})$ is sufficient to follow the rest of the paper.

Definition 1 (Simplicial complex). A simplicial complex is a pair $\mathcal{K}=(V, S)$, where $V$ is a finite set of vertices, and $S \subseteq \mathcal{P}(V)$ is a set of non-empty subsets of $V$ called simplices, such that:

- for every $v \in V,\{v\} \in S$, and

- $S$ is downward-closed, i.e., for every $Y \in S$ and for every non-empty subset $X \subseteq Y, X \in S$.

The dimension of a simplex $X \in S$ is defined by $\operatorname{dim}(X)=\operatorname{card}(X)-1$. We often identify a vertex $v \in V$ with the 0 -dimensional simplex $\{v\} \in S$. Simplexes of dimension 1 are called edges, and simplices of dimension 2 are triangles. The dimension of a simplicial complex $\mathcal{K}$ is the maximal dimension of a simplex of $\mathcal{K}$ : $\operatorname{dim}(\mathcal{K})=\max \{\operatorname{dim}(X) \mid X \in S\}$.

Definition 2 (Graph). A (non-directed, simple) graph $\mathcal{G}$ is a simplicial complex of dimension 1 . Such a graph is uniquely determined by its set $V$ of vertices and its set $E$ of edges, so we write $\mathcal{G}=(V, E)$.

We assume the reader is familiar with the notions of "cycle" and "induced subgraph". A graph is chordal when it has no induced cycle of length $\geq 4$. A clique of $G$ is a set of vertices $X \subseteq V$ such that for every $u, v \in X,\{u, v\} \in E$ is an edge of $\mathcal{G}$. The clique-number of $\mathcal{G}$, written $\omega(\mathcal{G})$, is the maximum number of vertices in a clique.

We denote by SimCpx the category of simplicial complexes (and simplicial maps), and Graph the category of graphs (and graph homomorphisms). Since we defined graphs as a special case of simplicial complexes, there is an inclusion functor $\iota:$ Graph $\rightarrow \operatorname{SimCpx}$. Conversely, there is a functor skel $1: \operatorname{SimCpx}_{1} \rightarrow$ Graph that can turn every simplicial complex into a graph (called its 1-skeleton) by forgetting about the higher-dimensional simplices. Formally, for a simplicial complex $\mathcal{K}=(V, S)$, its 1-skeleton is the graph $\operatorname{skel}_{1}(\mathcal{K})=(V, E)$ where $E=\{X \in S \mid \operatorname{dim}(X)=1\}$. Finally, we define a third functor $\kappa:$ Graph $\rightarrow$ SimCpx that associates with each graph $\mathcal{G}=(V, E)$ its complex of cliques, defined by $\kappa(\mathcal{G})=(V, S)$ where $S=\{X \subseteq V \mid X$ is a clique of $\mathcal{G}\}$.

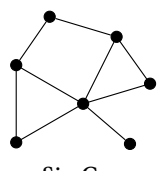

$\operatorname{SimCpx}$

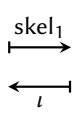

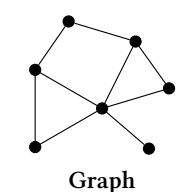

Graph
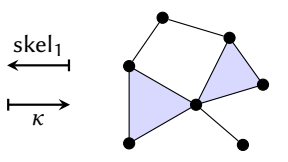

$\operatorname{SimCpx}$
The relationship between these three functors is nicely summed up in the following (folklore) proposition.

Proposition 1. The functor $\mathrm{l}$ is left adjoint to the 1-skeleton functor, and $\kappa$ is its right adjoint: $\iota \dashv \mathrm{skel}_{1} \dashv \kappa$. 
Finally, we mention the following topological characterization of chordal graphs in terms of contractibility.

THeorem 1 ([1]). A graph $\mathcal{G}$ is chordal if and only if for every induced subgraph $\mathcal{H}$ of $\mathcal{G}$, the complex of cliques $\kappa(\mathcal{H})$ is contractible.

Note that it is not true that a graph $\mathcal{G}$ is chordal if and only if $\kappa(\mathcal{G})$ is contractible: the counter-example below depicts a non-chordal graph (left) whose clique complex (right) is contractible, since it is homeomorphic to a 2-dimensional disc.
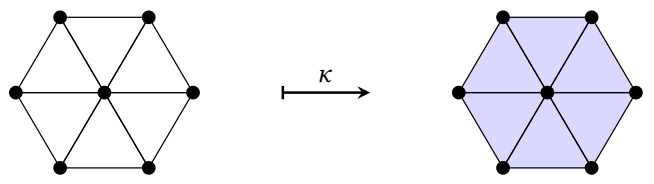

\section{GRAPH AGREEMENT TASKS AND SIMPLEX AGREEMENT}

We can now relate the three tasks defined in the introduction: edge agreement, clique agreement, and simplex agreement. In the statement below, when we say that two tasks are the same, we mean that they have the same set of values $\mathcal{V}$, and that their validity and agreement requirements are (respectively) equivalent.

Theorem 2. Let $\mathcal{G}=(V, E)$ be a graph. Then:

(1) Edge agreement on the graph $\mathcal{G}$ is the same task as simplex agreement on the simplicial complex $\iota(\mathcal{G})$.

(2) Clique agreement on $\mathcal{G}$ is the same task as simplex agreement on its simplicial complex of cliques, $\kappa(\mathcal{G})$.

Proof. By definition, both $\iota(\mathcal{G})$ and $\kappa(\mathcal{G})$ have the same set of vertices $V$ as the graph $\mathcal{G}$, therefore all four tasks use the same set of values $\mathcal{V}=V$. To prove (2), notice that a simplex of $\kappa(\mathcal{G})$ is by definition a clique of $\mathcal{G}$, so both validity and agreement conditions are the same. For (1), remark that a simplex of $\iota(\mathcal{G})$ can be either a vertex or an edge of $\mathcal{G}$. Once again, this observation makes the two validity and agreement conditions equivalent.

The purpose of Theorem 2 is to offer a new point of view on these two graph agreement tasks. While edge agreement is truly a graph task (because the complex $\iota(\mathcal{G})$ is 1 -dimensional), we argue that clique agreement is better understood when we look at the complex of cliques of the graph $\mathcal{G}$, to expose its topological nature. As an example, consider the following result of [12] about solving clique agreement for $n$ processes on a connected chordal graph $\mathcal{G}$, in a message-passing model with Byzantine faults:

Theorem 3 ([12]). For a chordal graph $\mathcal{G}$, clique agreement is solvable with $f$ Byzantine faults if $n>(\omega(\mathcal{G})+1) f$.

Now, observe that the simplicial complex $\kappa(\mathcal{G})$ is of dimension $\omega(\mathcal{G})-1$, where $\omega(\mathcal{G})$ denotes the clique number of $\mathcal{G}$. Thus, the above result can be compared with the one of Mendes et al. [11] for multidimensional agreement, which says that approximate agreement in $\mathbb{R}^{m}$ can be reached among $n$ processes with $f$ Byzantine faults if and only if $n>(m+2) f$. Hence, it appears that clique agreement behaves much like a discrete counterpart of the $m$-dimensional approximate agreement task, for $m=\operatorname{dim}(\kappa(\mathcal{G}))$.

A more recent paper [3] improved the solvability result of [12], in the wait-free model, to also work on some non-chordal graphs such as the hexagonal one depicted at the end of Section 2. They also proved an impossibility result: clique agreement is not waitfree solvable when the graph $\mathcal{G}$ satisfies a so-called lower bound labelling condition. This mysterious condition can be understood topologically: it implies that the complex of cliques $\kappa(\mathcal{G})$ is not simply connected. In fact, we conjecture that it is equivalent.

This topological point of view allows us to make the following conjecture, by analogy with the continuous case, where Herlihy and Shavit proved that $\varepsilon$-approximate agreement is impossible whenever the space $\mathcal{V} \subseteq \mathbb{R}^{m}$ contains holes of radius $\geq \varepsilon$ [9].

Conjecture. Simplex agreement on a simplicial complex $\mathcal{K}$ is wait-free solvable if and only if $\mathcal{K}$ is contractible.

Methods based on algebraic topology are well established in distributed computing to prove impossibility results [7]. In particular, the "only if" direction of the conjecture seems like it could be a direct consequence of the topological characterization of solvability for colorless tasks (also called convergence tasks in [4]), of which simplex agreement is an example.

For graphs, the conjecture implies that clique agreement on a graph $\mathcal{G}$ is solvable if and only if its complex of cliques $\kappa(\mathcal{G})$ is contractible. This is consistent with the solvability result of [12], since chordal graphs have a contractible complex of cliques by Theorem 1 . Our conjecture is also consistent with the impossibility result of [3], because graphs that admit a lower bound labelling have a non-simply connected complex of cliques (hence, non-contractible).

One case whose wait-free solvability is still unsettled is when the graph $\mathcal{G}$ is a triangulated sphere. For instance, take $\mathcal{G}$ to be the graph of edges of an icosahedron. Our conjecture says that, since the associated simplicial complex is not contractible, clique agreement on $\mathcal{G}$ should be unsolvable in the wait-free model.

\section{CONCLUSION}

We have shown that edge agreement and clique agreement both arise as particular cases of the simplex agreement task. While edge agreement is truly of a graph-theoretic nature, clique agreement appears to be better understood through the lens of simplicial complexes. With this new point of view, one can replace the graphtheoretic notions of "clique number" and "chordality" by the topological ones of "dimension" and "contractibility". With this in mind, parallels can be drawn with various results on multi-dimensional approximate agreement, which gives a better understanding on the wait-free solvability of graph agreement tasks.

All the tasks that we considered have a very permissive validity condition, where any output is allowed unless the input vertices already form a clique. By contrast, [12] and [3] have stronger validity conditions, requiring every output vertex to lie on a chordless or shortest path between the inputs. It would be insightful to study these conditions topologically, for example, to see whether they relate to notions of convex hull on manifolds.

\section{ACKNOWLEDGMENTS}

The author would like to thank Éric Goubault and Sergio Rajsbaum for helpful discussions on this topic. 


\section{REFERENCES}

[1] Karim A. Adiprasito, Eran Nevo, and Jose A. Samper. 2016. Higher chordality: From graphs to complexes. Proc. Amer. Math. Soc. 144, 8 (2016), 3317-3329. https://doi.org/10.1090/proc/13002

[2] Manuel Alcantara, Armando Castañeda, David Flores-Peñaloza, and Sergio Rajsbaum. 2019. The topology of look-compute-move robot wait-free algorithms with hard termination. Distributed Comput. 32, 3 (2019), 235-255. https://doi.org/10.1007/s00446-018-0345-3

[3] Dan Alistarh, Faith Ellen, and Joel Rybicki. 2021. Wait-free approximate agreement on graphs. (2021). arXiv:cs.DC/2103.08949

[4] Elizabeth Borowsky, Eli Gafni, Nancy A. Lynch, and Sergio Rajsbaum. 2001. The BG distributed simulation algorithm. Distributed Comput. 14, 3 (2001), 127-146. https://doi.org/10.1007/PL00008933

[5] Armando Castañeda, Sergio Rajsbaum, and Matthieu Roy. 2018. Convergence and covering on graphs for wait-free robots. F. Braz. Comput. Soc. 24, 1 (2018), 1:1-1:15. https://doi.org/10.1186/s13173-017-0065-8

[6] Danny Dolev, Nancy A. Lynch, Shlomit S. Pinter, Eugene W. Stark, and William E. Weihl. 1986. Reaching approximate agreement in the presence of faults. F. ACM 33, 3 (1986), 499-516. https://doi.org/10.1145/5925.5931
[7] Maurice Herlihy, Dmitry N. Kozlov, and Sergio Rajsbaum. 2013. Distributed Computing Through Combinatorial Topology. Morgan Kaufmann. https://store. elsevier.com/product.jsp?isbn=9780124045781

[8] Maurice Herlihy and Sergio Rajsbaum. 1997. The Decidability of Distributed Decision Tasks (Extended Abstract). In Proceedings of the Twenty-Ninth Annual ACM Symposium on the Theory of Computing, Frank Thomson Leighton and Peter W. Shor (Eds.). ACM, 589-598. https://doi.org/10.1145/258533.258652

[9] Maurice Herlihy and Nir Shavit. 1993. The asynchronous computability theorem for t-resilient tasks. In Proceedings of the Twenty-Fifth Annual ACM Symposium on Theory of Computing, S. Rao Kosaraju, David S. Johnson, and Alok Aggarwal (Eds.). ACM, 111-120. https://doi.org/10.1145/167088.167125

[10] Xingwu Liu, Zhiwei Xu, and Jianzhong Pan. 2009. Classifying rendezvous tasks of arbitrary dimension. Theor. Comput. Sci. 410, 21-23 (2009), 2162-2173. https: //doi.org/10.1016/j.tcs.2009.01.033

[11] Hammurabi Mendes, Maurice Herlihy, Nitin H. Vaidya, and Vijay K. Garg. 2015. Multidimensional agreement in Byzantine systems. Distributed Comput. 28, 6 (2015), 423-441. https://doi.org/10.1007/s00446-014-0240-5

[12] Thomas Nowak and Joel Rybicki. 2019. Byzantine Approximate Agreement on Graphs. In 33rd International Symposium on Distributed Computing, DISC 2019 (LIPIcs), Jukka Suomela (Ed.), Vol. 146. Schloss Dagstuhl - Leibniz-Zentrum für Informatik, 29:1-29:17. https://doi.org/10.4230/LIPIcs.DISC.2019.29 